\title{
New insights to the photometric structure of Blue Compact Dwarf Galaxies from deep near-infrared studies
}

\section{The sample of northern BCDs ${ }^{\star} \star \star$}

\author{
K. G. Noeske ${ }^{12}$, P. Papaderos ${ }^{1}$, L. M. Cairós ${ }^{1}$, and K. J. Fricke ${ }^{1}$ \\ 1 Universitäts-Sternwarte, Geismarlandstraße 11, 37083 Göttingen, Germany \\ e-mail: kai@ucolick.org \\ ${ }^{2}$ Lick Observatory, Univ. of California, 1156 High Street, Santa Cruz, CA 95064, USA
}

Received 7 February 2004 / Accepted 14 June 2004

\begin{abstract}
This paper is part of a series of publications which present a systematic study of Blue Compact Dwarf (BCD) Galaxies in the near infrared (NIR). Compared to the visible light, NIR data allow a better separation of the starburst emission from the light distribution of the old stellar low-surface brightness (LSB) host galaxy. We analyze deep NIR broad band images of a sample of $11 \mathrm{BCDs}$, observed with the Calar Alto ${ }^{\star \star \star} 3.6 \mathrm{~m}$ telescope. This work enlarges the samples presented in preceding papers of this study (Noeske et al. 2003, A\&A, 410, 481; Cairós et al. 2003, ApJ, 593, 312) by BCDs of the most common morphological type, displaying a regular elliptical LSB host galaxy. The data presented here allow the detection and quantitative study of the extended stellar LSB host galaxy in all sample BCDs. The NIR surface brightness profiles (SBPs) of the LSB host galaxies agree at large galactocentric radii with those from optical studies, showing also an exponential intensity decrease and compatible scale lengths. Similar to Noeske et al. (2003), we find centrally flattening exponential (type V) SBPs of the host galaxy for several BCDs. Such SBPs remain mostly undetected in optical bands, due to the comparatively stronger starburst emission at these wavelengths. We apply a modified exponential distribution to decompose and quantitatively analyze SBPs of LSB hosts with a type V intensity distribution. We present the results of the surface photometry and the decomposition of SBPs, and discuss individual objects with respect to morphological details of their star-forming regions.
\end{abstract}

Key words. galaxies: dwarf - galaxies: evolution - galaxies: structure - galaxies: starburst

\section{Introduction}

This paper is part of a series of publications which present a deep near infrared (NIR) imaging and surface photometry study of a large sample of Blue Compact Dwarf (BCD) Galaxies (Cairós et al. 2003, hereafter C03; Noeske et al. 2003, hereafter N03). As described in these papers, this project aims at an improved understanding of the structure and photometric properties of the old stellar low-surface brightness (LSB) host galaxy, and of the young stellar populations in BCDs. The LSB component contains practically all stellar mass of typical BCDs, and is therefore a likely dynamically important constituent of such galaxies (see references in N03). Its radial light

* Table 2 is also available in electronic form at the CDS via anonymous ftp to cdsarc.u-strasbg.fr $(130.79 .128 .5)$ or via http://cdsweb.u-strasbg.fr/cgi-bin/qcat?J/A+A/429/115

$\star \star$ Figures 2 to 11 are only available in electronic form at http://www.edpsciences.org

$\star \star \star$ German-Spanish Astronomical Center, Calar Alto, operated by the Max-Planck-Institute for Astronomy, Heidelberg, jointly with the Spanish National Commission for Astronomy. distribution, i.e. its projected luminosity density distribution, provides close constraints to the stellar mass distribution in a BCD, information which is crucial for modelling the global gravitational potential and dynamics of BCDs, as well as the effects of starburst events in such objects, such as galactic winds (e.g. De Young \& Heckman 1994; Mac Low \& Ferrara 1999; Silich \& Tenorio-Tagle 2001). The structural parameters (e.g., exponential scale length and central surface brightness) of the stellar LSB host also form a prime diagnostic tool for assessing the proposed evolutionary relations between different types of dwarf galaxies (see e.g. Lin \& Faber 1983; Thuan 1985; Dekel \& Silk 1986; Davies \& Phillipps 1988; Papaderos et al. 1996b, hereafter P96b; Marlowe et al. 1999; Dekel \& Woo 2003, see also the references in N03). Of equal interest are the relations between the properties of the stellar host galaxy and the occurrence and properties of starburst activity in BCDs (Loose \& Fricke 1982, P96b). The investigation of these latter issues requires a separation of the light distributions of the young and old stellar populations by means of a decomposition of radial surface brightness profiles (SBPs). Such analyses are however hampered by the extended dominant starburst emission at 
visible wavelengths, and can be performed with much better precision in the NIR (see N03, C03).

In the current paper, we present $J, H$ and $K^{\prime}$ image data and surface photometry of a sample of 11 BCDs, as well as a decomposition of the derived SBPs into the radial intensity distributions of the old LSB host galaxy and the starburst component. Morphological information is provided for each object, in particular for the star-forming (SF) regions. Remarkable features are briefly discussed. All data reduction and analysis procedures that were described in N03 have been unalteredly applied to the present data set.

Most of the objects in the present sample show an $\mathrm{iE} / \mathrm{nE}$ morphology, according to the classification scheme by Loose \& Thuan (1986, hereafter LT86). Such BCDs display SF regions either irregularly distributed (iE) or centrally confined $(\mathrm{nE})$ within a smooth elliptical or circular stellar LSB host galaxy. The $\mathrm{iE} / \mathrm{nE}$ BCDs comprise the majority ( 80-90\%) of the BCDs in the local Universe (LT86), but were underrepresented in the sample previously studied by N03 which contains mainly irregular morphological types and interacting objects. Earlier studies have indicated that the various morphological types of BCDs may systematically differ from each other in several physical properties (e.g. Salzer et al. 1989b; Telles et al. 1997; Noeske et al. 2000). For later comparisons of their NIR properties, a balanced representation of the different morphological types is therefore desirable. Analyses of the cumulative sample will be presented in forthcoming papers of the present series.

This paper is structured as follows: in Sect. 2, we list the subsample under study, and summarize the observations. In Sect. 3, some details concerning the derivation, analysis and decomposition of SBPs, described in depth in N03, are repeated to facilitate the understanding of this paper. This section also lists the results of the surface photometry, and of the decomposition of the surface brightness profiles, as well as colors of the old stellar host galaxies. Individual objects are presented and briefly discussed in Sect. 4, along with images, surface brightness profiles and color profiles. A brief summary is given in Sect. 5.

\section{Observations and data reduction}

The galaxies of this sample are listed in Table 1, along with their adopted distances, absolute $B$ magnitudes, Galactic extinction, and references to literature sources from which the latter values were taken. Selection criteria for distance determinations from the literature and details on the Galactic extinction values we adopt are given in Sects. 2.1 and 2.4.2 of N03, respectively.

The NIR images were observed with the $3.6 \mathrm{~m}$ telescope of the German-Spanish Astronomical Center, Calar Alto, Spain. Atmospheric conditions during the different observing runs were generally satisfactory (April 3rd, 1999: FWHM 1'.5, transparency average; December 26th, 1999: FWHM 1".3-3".5, transparency fair; May 10th-15th, 2000: FWHM 0.'8-1.'4, transparency good to average; October 6th-10th, 2000: FWHM 1'.2-2'.0, transparency good to fair). The telescope was equipped with the OMEGA PRIME camera, mounted at the prime focus. The $1024 \times 1024$ pixel Rockwell HAWAII detector of this instrument provided a pixel scale of 0.396 and a field of view of 6:76. Images were taken through the broad band filters $J$ and $H$, as well as the $K^{\prime}$ filter, which was preferred to a normal $K$ filter to attenuate the contribution of thermal background. Information on the observing technique and control of the time-dependent NIR background emission can be found in N03. The total on-object exposure times for each galaxy, after rejection of subexposures that were affected by unstable readout electronics or strong background gradients, are listed in Cols. 3-5 of Table 1. All data reduction procedures that were applied to the NIR images are detailed in N03. The angular resolution of the resulting images is listed in Col. 9 of Table 1.

\subsection{Flux calibration and extinction correction}

The transparency variations within each night, $\gtrsim 0.15$ mag in the $K^{\prime}$ band, did not allow a precise flux calibration through observations of standard stars. All data were therefore calibrated using fluxes of bright (typically $10 \ldots 15 \mathrm{~J}$ mag) field stars in the vicinity of the sample galaxies, given in the Two Micron All Sky Survey (2MASS) catalogue ${ }^{1}$ (see Cutri et al. 2000; Jarrett et al. 2000). Calibration errors for each galaxy are shown in Col. 14 of Table 2. These include uncertainties of 2MASS photometry for the field stars used for calibration, and measurement errors of these stars in our images. To calibrate the $K^{\prime}$ images, the 2MASS $K_{\mathrm{s}}$ fluxes were first transformed to $K^{\prime}$ magnitudes using Eqs. (3) from N03. The results listed within this paper therefore refer to the $J, H, K^{\prime}$ photometric system defined by the Calar Alto $3.6 \mathrm{~m}$ telescope and the OMEGA PRIME camera, tied to 2MASS zero points. Since color terms of the transformation between either photometric system are not available, intrinsic uncertainties of several 0.01 mag may be present. Notes on these uncertainties and on comparisons to data calibrated in other photometric systems are given in Sect. 2.4.1 of N03, and in Sect. 3.1 of this paper.

Magnitudes and colors given in this paper are corrected for Galactic extinction, adopting values (see Col. 7 of Table 1) derived from the $B$ band extinction maps by Schlegel et al. (1998) (cf. Table 1) and the standard $\left(R_{V}=3.1\right)$ extinction law (Cardelli et al. 1989) implemented into the NED. No attempt was made to correct for internal extinction, since this is known to vary spatially even in the most metal-deficient BCDs (cf., e.g., Guseva et al. 2001; Cannon et al. 2002) and can be reliably constrained in the SF regions only.

\section{Surface photometry and profile decomposition}

The SBPs and color profiles were derived as detailed in N03, using throughout the algorithm "iv" presented in Papaderos et al. (2002). A comparison to SBPs derived by alternative methods was performed for each profile to ensure consistency at both low and high $S / N$ levels. These comparison SBPs were generally obtained through methods "i" (ellipse fits to isophotes) and "iii" (area of all image pixels above a given

\footnotetext{
1 http://www.ipac.caltech.edu/2mass/
} 
Table 1. Sample galaxies; see Sect. 2.

\begin{tabular}{|c|c|c|c|c|c|c|c|c|c|}
\hline Object & $\begin{array}{l}\text { RA(J2000) } \\
\operatorname{Dec}(J 2000) \\
\text { (2) }\end{array}$ & $\begin{array}{l}{[\mathrm{s}]} \\
(3)\end{array}$ & $\begin{array}{l}{[s]} \\
(4)\end{array}$ & $\begin{array}{l}{[s]} \\
(5)\end{array}$ & $\begin{array}{c}M_{B} \\
\text { (ref.) } \\
{[\mathrm{mag}]} \\
(6)\end{array}$ & $\begin{array}{c}\text { [mag] } \\
(7)\end{array}$ & $\begin{array}{c}D \\
\text { (ref.) } \\
{[\mathrm{Mpc}]} \\
(8)\end{array}$ & $\begin{array}{c}F W H M \\
\text { (final) }^{*} \\
{\left[{ }^{\prime \prime}\right]} \\
(9)\end{array}$ & $\begin{array}{l}\text { Other } \\
\text { names }\end{array}$ \\
\hline $\begin{array}{l}\text { Mkn } 314 \\
\text { (iE) }\end{array}$ & $\begin{array}{r}23^{\mathrm{h}} 02^{\mathrm{m}} 59^{\mathrm{s}} .3 \\
+16^{\circ} 36^{\prime} 18^{\prime \prime} .9\end{array}$ & 1440 & 1740 & 1920 & $\begin{array}{c}-18.5 \\
\text { (f) }\end{array}$ & 0.38 & $\begin{array}{l}28.9 \\
\text { (a) }\end{array}$ & 1.5 & $\begin{array}{l}\text { NGC 7468; } \\
\text { UGC } 12329\end{array}$ \\
\hline $\begin{array}{l}\text { Mkn } 209 \\
\text { (iE) }\end{array}$ & $\begin{array}{r}12^{\mathrm{h}} 26^{\mathrm{m}} 16^{\mathrm{s}} .0 \\
+48^{\circ} 29^{\prime} 36^{\prime \prime} 6\end{array}$ & 1620 & 1440 & 1140 & $\begin{array}{c}-14.2 \\
(\mathrm{~g})\end{array}$ & 0.07 & $\begin{array}{l}5.8 \\
\text { (b) }\end{array}$ & 1.5 & $\begin{array}{l}\text { UGCA 281; I Zw 36; } \\
\text { Haro } 29\end{array}$ \\
\hline $\begin{array}{l}\text { Mkn } 996 \\
(\mathrm{nE})\end{array}$ & $\begin{array}{r}01^{\mathrm{h}} 27^{\mathrm{m}} 35^{\mathrm{s}} .5 \\
-06^{\circ} 19^{\prime} 36^{\prime \prime} 1\end{array}$ & 1260 & 1440 & 1200 & $\begin{array}{c}-16.6 \\
\text { (h) }\end{array}$ & 0.19 & $\begin{array}{l}20.4 \\
\text { (c) }\end{array}$ & 1.4 & \\
\hline $\begin{array}{l}\text { Mkn } 370 \\
(\mathrm{nE})\end{array}$ & $\begin{array}{r}02^{\mathrm{h}} 40^{\mathrm{m}} 29^{\mathrm{s}} .0 \\
+19^{\circ} 17^{\prime} 49^{\prime} .6\end{array}$ & 1140 & 1860 & 1640 & $\begin{array}{c}-17.1 \\
\text { (f) }\end{array}$ & 0.40 & $\begin{array}{l}11.2 \\
\text { (a) }\end{array}$ & 1.2 & $\begin{array}{l}\text { NGC 1036; IC 1828; } \\
\text { UGC } 2160\end{array}$ \\
\hline $\begin{array}{l}\text { I Zw } 115 \\
\text { (iI) }\end{array}$ & $\begin{array}{r}15^{\mathrm{h}} 32^{\mathrm{m}} 57^{\mathrm{s}} .0 \\
+46^{\circ} 27^{\prime} 06^{\prime \prime} .5 \\
\end{array}$ & 672 & 672 & 480 & $\begin{array}{c}-16.4 \\
(\mathrm{~g})\end{array}$ & 0.06 & $\begin{array}{l}15.0 \\
\text { (a) }\end{array}$ & 1.3 & $\begin{array}{l}\text { UGC 9893; } \\
\text { VV } 720\end{array}$ \\
\hline $\begin{array}{l}\text { Mkn } 5 \\
\text { (iE/iI, C) }\end{array}$ & $\begin{array}{r}06^{\mathrm{h}} 42^{\mathrm{m}} 15^{\mathrm{s}} .5 \\
+75^{\circ} 37^{\prime} 32^{\prime \prime} .6\end{array}$ & 1650 & 2100 & 2340 & $\begin{array}{c}-15.7 \\
\text { (f) }\end{array}$ & 0.36 & $\begin{array}{l}15.3 \\
\text { (a) }\end{array}$ & 1.5 & UGCA 130 \\
\hline $\begin{array}{l}\text { Mkn } 600 \\
\text { (iE) }\end{array}$ & $\begin{array}{r}02^{\mathrm{h}} 51^{\mathrm{m}} 04^{\mathrm{s}} .6 \\
+04^{\circ} 27^{\prime} 13^{\prime \prime} .9 \\
\end{array}$ & 1560 & 1320 & 1080 & $\begin{array}{c}-15.5 \\
\text { (f) }\end{array}$ & 0.28 & $\begin{array}{l}12.6 \\
\text { (a) }\end{array}$ & 1.1 & \\
\hline $\begin{array}{l}\text { NGC } 6789 \\
\text { (iE) }\end{array}$ & $\begin{array}{r}19^{\mathrm{h}} 16^{\mathrm{m}} 41^{\mathrm{s}} .9 \\
+63^{\circ} 58^{\prime} 18^{\prime \prime} .0\end{array}$ & 1200 & 1680 & 1920 & $\begin{array}{c}-14.3 \\
\text { (i) }\end{array}$ & 0.30 & $\begin{array}{l}3.6 \\
\text { (d) }\end{array}$ & 1.4 & UGC 11425 \\
\hline $\begin{array}{l}\text { Mkn } 324 \\
\text { (iE) }\end{array}$ & $\begin{array}{r}23^{\mathrm{h}} 26^{\mathrm{m}} 32^{\mathrm{s}} .8 \\
+18^{\circ} 15^{\prime} 59^{\prime} .8 \\
\end{array}$ & 1680 & 600 & 1140 & $\begin{array}{c}-16.5 \\
\text { (f) }\end{array}$ & 0.22 & $\begin{array}{l}21.8 \\
\text { (a) }\end{array}$ & 1.4 & UGCA 439 \\
\hline $\begin{array}{l}\text { Mkn } 450 \\
\text { (iE) }\end{array}$ & $\begin{array}{r}13^{\mathrm{h}} 14^{\mathrm{m}} 48^{\mathrm{s}} .3 \\
+34^{\circ} 52^{\prime} 51^{\prime \prime} .3 \\
\end{array}$ & 1200 & 1440 & 1920 & $\begin{array}{c}-16.7 \\
(\mathrm{j})\end{array}$ & 0.06 & $\begin{array}{l}17.9 \\
\text { (a) }\end{array}$ & 1.3 & $\begin{array}{l}\text { UGC 08323; VV 616; } \\
\text { HS 1312+3508 }\end{array}$ \\
\hline $\begin{array}{l}\text { NGC } 5058 \\
\text { (iI?) }\end{array}$ & $\begin{array}{r}13^{\mathrm{h}} 16^{\mathrm{m}} 52^{\mathrm{s}} .3 \\
+12^{\circ} 32^{\prime} 53^{\prime \prime} .9\end{array}$ & 720 & 1680 & 1440 & $\begin{array}{l}-15.9 \\
\text { (i) }\end{array}$ & 0.13 & $\begin{array}{l}11.6 \\
(\mathrm{c}, \mathrm{e})\end{array}$ & 1.1 & $\begin{array}{l}\text { UGC 08345; Mkn 786; } \\
\text { KPG } 370\end{array}$ \\
\hline
\end{tabular}

* Resolution of the best image set available for the respective galaxy, after reduction and combination; (a) Tully (1988); (b) Schulte-Ladbeck et al. (2001); (c) inferred from the heliocentric velocity $v_{\text {hel }}$ listed in the NED, corrected for solar motion with respect to the center of the Virgo Cluster and adopting $H_{0}=75 \mathrm{~km} \mathrm{~s}^{-1} \mathrm{Mpc}^{-1}$ (cf. N03); (d) Drozdovsky et al. (2001); (e) see Sect. 4.11; (f) $m_{B}$ from Table 2 in Cairós et al. (2001a); (g) $m_{B}$ from Papaderos et al. (1996a); (h) $m_{B}$ from Thuan et al. (1996); (i) $m_{B}$ from the RC3 (de Vaucouleurs et al. 1991); (j) $m_{B}$ from Vennik et al. (2000).

intensity threshold) described in Papaderos et al. (1996a, hereafter P96a).

As described in N03, uncertainties as displayed for each SBP are very conservative upper limits to the true uncertainties and are likely often overestimated.

Also the decomposition of the derived SBPs into the intensity distributions of the LSB host and the starburst component, which is required to separately study the emission of either stellar component, is explained in Sect. 3.2 of N03.

For a better understanding of the results of the present work (Table 2), we reiterate here the functions that were employed to fit the SBPs of the LSB host galaxies. The resulting parameters of those fits provide the structural parameters of the LSB host, and make it possible to calculate a model of its intensity distribution, which is then subtracted from the total SBPs to obtain the light distribution of the starburst component.

At large galactocentric radii ( $>2 \ldots 3$ scale lengths $\alpha$ ), the SPBs of the LSB hosts show for most BCDs a smooth decay, which can typically be approximated by an exponential law (e.g. LT86, P96a, Telles et al. 1997; Cairós et al. 2001a, hereafter C01a).

If the intensity is expressed in terms of the surface brightness $\mu$, this function reads as

$\mu\left(R^{\star}\right)=\mu_{\mathrm{E}, 0}+1.0857 \frac{R^{\star}}{\alpha}$

with $\mu_{\mathrm{E}, 0}, \alpha$ and $R^{\star}$ denoting, respectively, the extrapolated central surface brightness in mag/ $\square^{\prime \prime}$, the exponential scale length, and the photometric radius.

As detailed in N03, an exponential fit to the outer SBP of the LSB host provided in some cases no meaningful decomposition, but exceeded the intensity of the observed total SBP at small radii. In these cases, where a central flattening of the outer exponential intensity distribution of the LSB host ("type V SBP", Binggeli \& Cameron 1991, see Sect. 3.2 in N03) had to be postulated, a modified 
Table 2. Structural properties of the $\operatorname{dwarfs}^{a}$; see Sect. 3 for explanations. See also discussions of individual objects.

\begin{tabular}{|c|c|c|c|c|c|c|c|c|c|c|c|c|c|}
\hline $\begin{array}{l}\text { Name } \\
(b, q)^{b}\end{array}$ & Band & $\begin{array}{c}\left(\mu_{E, 0}\right)^{c} \\
\mathrm{mag} / \square^{\prime \prime}\end{array}$ & $\begin{array}{c}\alpha \\
\mathrm{kpc}\end{array}$ & $\begin{array}{c}m_{\mathrm{LSB}}^{\mathrm{fit}} \\
\text { mag }\end{array}$ & $\begin{array}{l}P_{\text {iso }} \\
\mathrm{kpc}\end{array}$ & $\begin{array}{l}m_{P_{\text {iso }}} \\
\text { mag }\end{array}$ & $\begin{array}{l}E_{\text {iso }} \\
\mathrm{kpc}\end{array}$ & $\begin{array}{r}m_{\mathrm{E}_{\text {iso }}} \\
\text { mag }\end{array}$ & $\begin{array}{r}m_{\mathrm{SBP}} \\
\mathrm{mag}\end{array}$ & $\begin{array}{c}\left(m_{\mathrm{tot}}\right)^{c} \\
\text { mag }\end{array}$ & $\begin{array}{c}r_{\mathrm{eff}}, r_{80} \\
\mathrm{kpc}\end{array}$ & $\eta_{\mathrm{SBP}}$ & $\begin{array}{l}\sigma_{\text {cal }} \\
\text { mag }\end{array}$ \\
\hline (1) & (2) & (3) & (4) & (5) & (6) & (7) & (8) & (9) & 0) & (11) & (12) & (13) & 4) \\
\hline Mkn 314 & $J$ & $19.10 \pm 0.13$ & $00 \pm 0.04$ & 12.84 & 1.85 & 12.84 & 3.58 & 12.99 & 12.12 & $12.05 \pm 0.01$ & $0.88,1.86$ & 1.46 & 0.03 \\
\hline \multirow[t]{2}{*}{$\star^{f}$} & $H$ & $18.61 \pm 0.15$ & $1.09 \pm 0.05$ & 12.17 & 1.70 & 12.26 & 3.39 & 12.39 & 11.51 & $11.38 \pm 0.01$ & $0.90,1.96$ & 1.50 & 0.03 \\
\hline & $K^{\prime}$ & $.73 \pm 0.17$ & $22 \pm 0.07$ & 12.04 & 2.12 & 11.77 & 3.67 & 12.28 & 11.21 & $11.17 \pm 0.02$ & .88 & 1.45 & 0.03 \\
\hline Mkn 209 & $J$ & $20.21 \pm 0.06$ & $0.23 \pm 0.01$ & 13.63 & 0.16 & 16.37 & 0.60 & 13.98 & 13.69 & $13.48 \pm 0.01$ & $0.29,0.47$ & 1.69 & 0.03 \\
\hline \multirow[t]{2}{*}{$\star^{f}$} & $H^{d, i}$ & - & - & - & - & - & - & - & 13.38 & $13.15 \pm 0.01$ & $0.29,0.45$ & 1.31 & 0.06 \\
\hline & $K^{\prime h}$ & - & - & - & - & - & - & - & 13.10 & $13.01 \pm 0.06$ & $0.28,0.44$ & 1.37 & 0.07 \\
\hline Mkn 996 & $J$ & $18.20 \pm 0.03$ & $0.40 \pm 0.01$ & 13.17 & 0.67 & 14.50 & 1.77 & 13.24 & 12.91 & $12.87 \pm 0.01$ & $0.51,1.01$ & 1.53 & 0.03 \\
\hline \multirow[t]{2}{*}{$\star$} & $H$ & $17.80 \pm 0.04$ & $0.42 \pm 0.01$ & 12.65 & 0.69 & 13.74 & 1.64 & 12.76 & 12.33 & $12.26 \pm 0.01$ & $0.51,1.03$ & 1.67 & 0.04 \\
\hline & $K^{\prime e}$ & $17.48 \pm 0.05$ & $0.43 \pm 0.01$ & 12.32 & 0.68 & 13.20 & 1.77 & 12.41 & 12.04 & $11.94 \pm 0.02$ & $0.42,0.84$ & 1.90 & 0.05 \\
\hline $\mathrm{Mkn}$ & $J$ & 10.0010 .00 & $0.64 \pm 0.01$ & 11.52 & 0.80 & 12.80 & 2.43 & 11.64 & 11.29 & $11.27 \pm 0.01$ & $0.72,1.49$ & 1.68 & 0.03 \\
\hline \multirow[t]{2}{*}{$\star^{g}$} & $H$ & $18.36 \pm 0.06$ & $0.63 \pm 0.01$ & 11.04 & 0.84 & 12.14 & 2.12 & 11.22 & 10.74 & $10.72 \pm 0.04$ & $0.71,1.46$ & 1.72 & 0.07 \\
\hline & $K^{\prime}$ & $18.00 \pm 0.22$ & $0.59 \pm 0.04$ & 10.83 & 0.78 & 12.03 & 2.17 & 10.97 & 10.57 & $10.46 \pm 0.03$ & $0.67,1.39$ & 1.55 & 0.05 \\
\hline IZw 115 & $J$ & $19.52 \pm 0.13$ & $0.53 \pm 0.02$ & 13.68 & 0.85 & 15.05 & 1.68 & 14.01 & 13.46 & $13.26 \pm 0.02$ & .46 & 1.15 & 0.04 \\
\hline \multirow[t]{2}{*}{$(2.3,0.8)$} & $H$ & $19.23 \pm 0.35$ & $0.56 \pm 0.06$ & 13.25 & 0.80 & 14.49 & 1.35 & 13.91 & 0 & 97 & 6 & 5 & 66 \\
\hline & $K^{\prime h}$ & - & - & - & - & - & - & - & 12 & $12.64 \pm 0.07$ & 32 & 1.13 & 0.08 \\
\hline Mkn 5 & $J$ & $19.03 \pm 0.04$ & $0.37 \pm 0.01$ & 13.73 & 0.54 & 15.90 & 1.35 & 13.90 & 13.73 & $13.58 \pm 0.01$ & $0.56,0.92$ & 1.08 & 0.03 \\
\hline \multirow[t]{2}{*}{$(1.4,0.65)$} & $H$ & $18.78 \pm 0.07$ & $0.40 \pm 0.01$ & 13.30 & 0.58 & 14.94 & 1.18 & 13.56 & 13.21 & $13.16 \pm 0.02$ & 0.92 & 1.04 & 0.04 \\
\hline & $K^{\prime}$ & $18.41 \pm 0.06$ & $0.40 \pm 0.01$ & 12.92 & 0.56 & 14.86 & 1.33 & 13.14 & 12.93 & $12.78 \pm 0.02$ & $0.57,0.91$ & 1.22 & 0.05 \\
\hline Mkn 600 & $J$ & $19.35 \pm 0.08$ & $0.30 \pm 0.01$ & 13.93 & 0.41 & 15.63 & 0.99 & 14.11 & 13.86 & $13.78 \pm 0.04$ & $0.35,0.64$ & 1.34 & 0.05 \\
\hline \multirow[t]{2}{*}{$\star^{f}$} & $H$ & $19.09 \pm 0.08$ & $0.32 \pm 0.01$ & 13.48 & 0.40 & 15.07 & 0.87 & 13.79 & 13.41 & $13.23 \pm 0.07$ & $0.36,0.66$ & 1.35 & 0.07 \\
\hline & $K^{\prime d}$ & $19.01 \pm 0.25$ & $0.32 \pm 0.04$ & 13.42 & 0.43 & 14.67 & 0.88 & 13.72 & 13.25 & $13.13 \pm 0.07$ & $0.35,0.65$ & 1.52 & 0.06 \\
\hline NGC & $J$ & 1 & 0 & 11.93 & 0 & 1 & 0 & 1 & 2 & 03 & 57 & 1.07 & 0.03 \\
\hline \multirow[t]{2}{*}{$(3.3,0.70)$} & $H$ & $18.37 \pm 0.08$ & $0.20 \pm 0.01$ & 11.51 & 0.25 & 13.56 & 0.64 & 11.78 & 11.38 & $11.32 \pm 0.03$ & $0.33,0.56$ & 1.13 & 0.03 \\
\hline & $K^{\prime}$ & $18.12 \pm 0.32$ & $0.20 \pm 0.02$ & 11.25 & 0.23 & 13.58 & 0.69 & 11.48 & 11.19 & $11.08 \pm 0.04$ & $0.34,0.57$ & 1.26 & 0.02 \\
\hline $\mathrm{Mk}$ & $J$ & $17.81 \pm 0.03$ & $0.28 \pm 0.0$ & 13.68 & 0 & 1 & 1 & 3 & 13.32 & $13.25 \pm 0.01$ & 7 & 1.26 & 0.04 \\
\hline \multirow[t]{2}{*}{$\star$} & $H$ & $17.36 \pm 0.10$ & $0.29 \pm 0.01$ & 13.17 & 0.55 & 14.08 & 1.24 & 13.26 & 12.81 & $12.75 \pm 0.02$ & $0.34,0.68$ & 1.30 & 0.04 \\
\hline & $K^{\prime d}$ & $16.93 \pm 0.12$ & $0.26 \pm 0.01$ & 12.94 & 0.50 & 14.07 & 1.24 & 13.00 & 12.66 & $12.55 \pm 0.02$ & $0.32,0.62$ & 1.23 & 0.05 \\
\hline $\mathrm{Mkn}$ & $J$ & $19.91 \pm 0.04$ & $0.87 \pm 0.01$ & 13.09 & 1.41 & 14.41 & 2.48 & 13.43 & 12.90 & $12.80 \pm 0.01$ & $1.25,2.08$ & 1.46 & 0.05 \\
\hline \multirow[t]{2}{*}{$(1.5,0.65)$} & $H^{d}$ & $19.17 \pm 0.13$ & $0.80 \pm 0.03$ & 12.55 & 1.18 & 14.18 & 2.08 & 12.96 & 12.37 & $12.35 \pm 0.01$ & $1.22,2.01$ & 1.06 & 0.07 \\
\hline & $K^{\prime h}$ & - & - & - & - & - & - & - & 12.16 & $12.05 \pm 0.02$ & $1.20,1.99$ & 1.27 & 0.05 \\
\hline NGC 5058 & $J$ & $19.08 \pm 0.13$ & $0.47 \pm 0.02$ & 12.47 & 0.72 & 13.67 & 1.70 & 12.62 & 12.21 & $12.18 \pm 0.01$ & $0.56,1.13$ & 1.44 & 0.05 \\
\hline \multirow[t]{2}{*}{$\star^{f}$} & $H$ & 18. & $0.52 \pm 0.01$ & 12.14 & 0.80 & 12.71 & 1.45 & 12.42 & 11.65 & $11.67 \pm 0.02$ & $0.55,1.10$ & 1.42 & 0.07 \\
\hline & $K^{\prime}$ & $18.62 \pm 0.14$ & $9 \pm 0.02$ & 11.94 & 0.79 & 12.56 & 1.52 & 12.15 & 11.49 & $11.48 \pm 0.03$ & $0.52,1.02$ & 1.46 & 0.07 \\
\hline
\end{tabular}

${ }^{a}$ All values are corrected for Galactic extinction, adopting the $A_{B}$ from Table 1.

${ }^{b}$ See Sect. 3 for details. Objects for which the LSB component was modelled by a pure exponential (Eq. (1)) are marked with an asterisk.

${ }^{c}$ Errors do not include the calibration uncertainties given in Col. 14.

${ }^{d}$ Decomposition less reliable in this filter (low $S / N$, or data affected by nearby bright stars).

${ }^{e} K^{\prime}$ SBP less deep than $J$ and $H$; effects on Cols. 10, 11 and 13.

${ }^{f}$ Small systematic deviations from an exponential LSB profile, likely type V SBP (see notes of caution in Sect. 3 ).

${ }^{g}$ Small systematic deviations from an exponential LSB profile, possible type V SBP (see Sect. 4.4, and notes of caution in Sect. 3).

${ }^{h}$ Detected or likely type V SBP restricts exponential part of LSB fit to low $S / N$ region, preventing robust decomposition in the $K$ band.

${ }^{i}$ Likely type V SBP, cannot be reliably modelled; exponential LSB fit yields unphysical $H$ band decomposition (see Sect. 4.2). 
exponential distribution Eq. (2) was applied (cf. P96a) to decompose the total SBPs:

$I\left(R^{\star}\right)=I_{\mathrm{E}, 0} \exp \left(-\frac{R^{\star}}{\alpha}\right)\left\{1-q \exp \left(-P_{3}\left(R^{\star}\right)\right)\right\}$

with

$P_{3}\left(R^{\star}\right)=\left(\frac{R^{\star}}{b \alpha}\right)^{3}+\left(\frac{R^{\star}}{\alpha} \cdot \frac{1-q}{q}\right)$.

This empirical function, in the following referred to as med, flattens with respect to a pure exponential law inside a cutoff radius $b \alpha$, and attains at $R^{*}=0^{\prime \prime}$ an intensity given by the relative depression parameter $q=\Delta I / I_{\mathrm{E}, 0}<1$, where $I_{\mathrm{E}, 0}$ is the extrapolated central intensity of the unflattened exponential. Details on the determination of the parameters of the med, $\mu_{\mathrm{E}, 0}, \alpha$, and $(b, q)$, can be found in Sect. 3.2 of N03.

For several objects, the SBPs of the LSB host show small systematic deviations from an exponential slope, yet at low significance levels. Confirmation or reliable quantification of a type V SBP was not possible, so these galaxies were decomposed by means of exponential fits. Nevertheless, type V SBPs are likely for Mkn 209, Mkn 314, Mkn 600 and NGC 5058, and possible for Mkn 370. If type V SBPs of the LSB host are present in these galaxies, the decompositions by means of exponential fits will be imprecise, as detailed in N03. Most importantly, the resulting host galaxy flux will be overestimated, and the starburst component correspondingly underestimated.

Alternative fits to the light distribution of the LSB host using the Sérsic law were abandoned in the present work. As detailed in $\mathrm{N03}$ and $\mathrm{C} 03$, the use of the latter empirical function to model the LSB emission of BCDs entails several problems and, as it demands a currently unavailable data quality, frequently does not yield a robust solution. No LSB host showed a SBP that gave signs of being steeper than an exponential (Sérsic index $\eta>1$ ).

Sérsic fits were merely used to describe the total SBPs. In the case of BCDs, total SBPs include the emission of both the starburst component and the LSB host galaxy, whose mass-tolight ratios differ strongly from each other. These Sérsic fits are therefore of limited use to understand the physics of BCDs, and were applied to allow comparisons with previous surface photometry studies where such fits were performed, and to high-redshift objects for which only integral profiles can be derived.

The photometric quantities that were derived for the sample BCDs are summarized in Table 2. BCDs for which a type V profile in their underlying LSB component was either not detected, or could not be reliably confirmed and modelled (see above), were decomposed by means of a pure exponential (Eq. (1)) and are marked with an asterisk in Col. 1. For the remaining systems we list the $(b, q)$ parameters, as obtained by fitting Eq. (2) to SBPs of the LSB host galaxy. The latter SBPs were derived from images out of which irregular starburst emission had been largely removed (N03). Columns 3 and 4 list, respectively, the extrapolated central surface brightness $\mu_{\mathrm{E}, 0}$ and the exponential scale length $\alpha$, obtained by fitting an exponential (Eq. (1)) to the outer exponential LSB part of each SBP.
Column 5 gives the total apparent magnitude of the LSB component, computed by extrapolating the fitted model (i.e. either an exponential or a med) to $R^{\star}=\infty$. Columns 6 through 9 list the radii and magnitudes of the star-forming $(\mathrm{P})$ and underlying stellar LSB component (E), as obtained by profile decomposition. Following P96a, we measure the respective radial extent $\left(P_{\text {iso }}, E_{\text {iso }}\right)$ and encircled magnitude $\left(m_{P_{\text {iso }}}, m_{E_{\text {iso }}}\right)$ of each component at an isophotal level iso, taken to be $23 \mathrm{mag} / \square^{\prime \prime}$ for $J$ and $22 \mathrm{mag} / \square^{\prime \prime}$ for $H$ and $K^{\prime}$. The isophotal radii determined for the sample BCDs at $23 J \mathrm{mag} / \square^{\prime \prime}$ turn out to be comparable to those obtained from optical SBPs at $25 \mathrm{Bmag} / \square^{\prime \prime}$ ( $P_{25}$ and $E_{25}$ in P96a). Column 10 lists the magnitude from an SBP integration out to the last data point, and total magnitudes from aperture measurements (cf. Sect. 3.2) are listed in Col. 11. The radii $r_{\text {eff }}$ and $r_{80}$, enclosing $50 \%$ and $80 \%$ of the SBP's flux are included in Col. 12. Finally, a formal Sérsic exponent for the whole $\mathrm{SBP}\left(\eta_{\mathrm{SBP}}\right)$, for later comparison with literature data, is listed in Col. 13 of Table 2. Column 14 gives the $J, H, K^{\prime}$ calibration uncertainties for each galaxy (Sect. 2.1).

\subsection{Colors of the underlying LSB host galaxy}

Colors of the LSB host galaxy were derived as the errorweighted mean of the color profiles, outside radii affected by SF activity (cf. Sect. 3.5. in N03). Deviant points, being probably affected by uncertainties in the sky determination, and local residuals from the subtraction of background sources were rejected. The mean colors of the host galaxies are shown at the right edge of each color profile (Figs. 1-??). Table 3 lists the resulting colors, in the photometric system described in Sect. 2.1. $H-K^{\prime}$ colors given in Col. 3 were transformed to $H-K_{\mathrm{s}}$ to facilitate comparisons to other photometric systems. Colors in Table 3 can therefore be considered 2MASS colors. The relatively large errors in Cols. 2 and 3 include conservative upper limits to all uncertainties that would apply when comparing those values to other colors in the 2MASS system. These are: (i) zeropoint uncertainties (Table 2, Col. 14); (ii) the rms scatter of the averaged color profile points, as upper limit to effects of local background instabilities and fore/background sources; (iii) an estimate of the systematic uncertainties of SBP derivation in the low $S / N$ regime $(0.05,0.07$ and $0.1 \mathrm{mag}$ for good, medium and low $S / N$ data, respectively); (iv) an upper limit $(0.08 \mathrm{mag}$, cf. N03, Sect. 2.4.1) of possible uncertainties from unknown color term transformations between our filters and those used by $2 \mathrm{MASS}$; (v) an additional uncertainty estimate of $0.05 \mathrm{mag}$ in $H-K_{\mathrm{s}}$ to account for the $K^{\prime}$ to $K_{\mathrm{s}}$ transformation.

\subsection{Unsharp masking technique and aperture photometry}

To improve on studies of fine morphological details, the hierarchical binning $(\mathrm{hb})$ transformation, a modified unsharp masking technique described in e.g. Papaderos (1998), was applied to the data. Features of interest are displayed in the insets of Figs. 1-??. 
Table 3. Colors of the host galaxy ${ }^{a}$.

\begin{tabular}{lcc}
\hline \hline Object & $\begin{array}{c}J-H \\
{[\mathrm{mag}]}\end{array}$ & $\begin{array}{c}H-K_{\mathrm{s}} \\
{[\mathrm{mag}]}\end{array}$ \\
\hline Mkn 314 & $0.77 \pm 0.16^{b}$ & $0.26 \pm 0.18^{b}$ \\
Mkn 209 & $0.37 \pm 0.13^{b}$ & $0.19 \pm 0.18^{b}$ \\
Mkn 996 & $0.56 \pm 0.13$ & $0.37 \pm 0.16^{b}$ \\
Mkn 370 & $0.52 \pm 0.14$ & $0.16 \pm 0.17$ \\
IZw 115 & $0.46 \pm 0.15^{b}$ & $0.31 \pm 0.26^{b}$ \\
Mkn 5 & $0.54 \pm 0.15^{b}$ & $0.45 \pm 0.12^{b}$ \\
Mkn 600 & $0.50 \pm 0.17^{b, c}$ & $0.08 \pm 0.29^{b, c}$ \\
NGC 6789 & $0.42 \pm 0.12$ & $0.31 \pm 0.18^{b}$ \\
Mkn 324 & $0.55 \pm 0.12$ & $0.22 \pm 0.18^{b}$ \\
Mkn 450 & $0.51 \pm 0.16$ & $0.19 \pm 0.26^{b}$ \\
NGC 5058 & $0.41 \pm 0.16$ & $0.24 \pm 0.25^{b}$ \\
\hline
\end{tabular}

Colors can be directly compared to data in the 2MASS photometric system; for details of the photometric system and the various error sources that are considered here, see Sects. 3.1 and 2.1.

${ }^{a}$ Corrected for galactic extinction (see Sect. 2.1).

${ }^{b}$ Possible local instabilities in one SBP at low $S / N$ levels.

${ }^{c}$ Possible contamination by nebular emission over a large portion of the LSB host galaxy.

Total magnitudes of the galaxies were derived within polygonal apertures which extend typically out to 1.5 Holmberg radii (Col. 11 of Table 2), after removal of foreand background sources from the area of interest (cf. Sect. 2). These magnitudes can generally be considered more accurate than those inferred from an integration of SBPs (see N03).

\section{Results and discussion of individual objects}

\subsection{Mkn 314 (NGC 7468, UGC 12329)}

As an iE-classified system, Mkn 314 represents the most common morphological BCD type (LT86). Optical surface photometry as well as deep $\mathrm{H} \alpha$ imaging have been presented in C01a and Cairós et al. (2001b, hereafter C01b). This intrinsically luminous $\mathrm{BCD}\left(M_{B}=-18.5\right)$ was morphologically selected as a candidate polar-ring galaxy (Whitmore et al. 1990; van Driel et al. 2001), and was included in the study of Markarian galaxies with multiple nuclei by Mazzarella \& Boroson (1993). Optical broad-band images by the latter authors revealed three prominent maxima, roughly arranged along the major axis of the galaxy (labeled a through $c$ in Fig. 1a). Nordgren et al. (1995) found that regions a and c, situated at comparable projected distances $(\approx 10 . ' 5$ or $0.57 \mathrm{kpc}$ and 9.4 or $0.52 \mathrm{kpc}$, respectively) from the optically brightest, central source b, show velocity differences to the latter of $30 \pm 17$ and $20 \pm 14 \mathrm{~km} \mathrm{~s}^{-1}$. These values are of the order of typical H I velocity dispersions in BCDs (van Zee et al. 1998), and do not provide any strong evidence for kinematical distortions which might indicate a previous merger event. Narrow band images reveal that SF activity in the galaxy is distributed along the northeast-southwest direction, in a bar-like structure, which extends to about $5 \mathrm{kpc}$ southwest from the nuclear region (Deeg et al. 1997, C01b). BCDs showing such bar-structures are relatively frequent (for instance Mkn 370, this paper; Mkn 35, II Zw 71, C01b; II Zw 33, Walter et al. 1997) and are morphologically classified as "chain starburst" in $\mathrm{C} 01 \mathrm{~b}$. The total $\mathrm{H} \alpha$ luminosity of the galaxy amounts to $\sim 6.1 \times 10^{40} \mathrm{erg} \mathrm{s}^{-1}$ ( $\mathrm{C} 01 \mathrm{~b}$, for the distance adopted here). In the $\mathrm{H} \alpha$ frames, three strong SF regions are detected, aligned with the central sources detected in the broad-band frames. The peak of the $\mathrm{H} \alpha$ emission is located at knot $\mathrm{c}$, whereas $\mathrm{b}$ and a are moderate sources.

The morphologies in the optical and in the NIR basically coincide, though the NIR frames provide a better spatial resolution. Smaller condensations, surrounding the latter three major sources, were identified (named $d-h$ in Fig. 1a).

The starburst population is immersed in an extended older population of stars, which displays elliptical isophotes and red colors $(B-R \approx 1, \mathrm{C} 01 \mathrm{ab})$. Since the chain of SF sources along the major axis of Mkn 314 is less prominent in the NIR than in optical wavelengths (cf. C01b), slight differences in the outer slope of optical and NIR SBPs of the LSB component are to be expected. In $J$ and $H$ we derive a scale length of $\sim 1 \mathrm{kpc}$, somewhat smaller than the optical value $(\sim 1.2 \mathrm{kpc}, \mathrm{C} 01 \mathrm{a})$. NIR SBPs reveal a slight curvature for $R^{*} \geq 20^{\prime \prime}$ pointing to a type V SBP; however, the $S / N$ level in this outermost region is too low to corroborate this conclusion.

A tail structure, formed by several SF knots, departs from the central regions to the south-west, connecting with an extended source named "S1" in Fig. 1a. This object, which we detect in all NIR bands, splits into two compact $\mathrm{H}$ II regions on $\mathrm{H} \alpha$ frames (C01b). The blue optical colors $(B-R \sim 0.5 \mathrm{mag}$; $\mathrm{C} 01 \mathrm{~b})$ of $\mathrm{S} 1$ are comparable to those observed in the central part of the BCD (regions a through c, C01b). From the present data we cannot judge whether $\mathrm{S} 1$ is a gaseous or stellar interloper infalling into Mkn 314: the projected velocity difference of $30 \mathrm{~km} \mathrm{~s}^{-1}$ (Nordgren et al. 1995) to Mkn 314 is not unusual for close dwarf companions of BCDs (Noeske et al. 2001a). Also, Taylor et al. (1994) find from interferometric H I observations of Mkn 314 little indication for an interaction and describe merely an "oval distortion" of the gaseous component.

\subsection{Mkn 209 (UGCA 281, I Zw 36, Haro 29)}

Mkn 209 provides an example of an intrinsically faint $\left(M_{B}=\right.$ -14.2), compact (optical radius of $\approx 0.6 \mathrm{kpc}$ at $25 \mathrm{Bmag} / \square^{\prime \prime}$, P96a) and metal-deficient $\left(Z \approx Z_{\odot} / 14\right.$, Izotov \& Thuan 1999) BCD with an iE-morphology (LT86). This galaxy has been the subject of numerous studies, due to its relative proximity $(D \approx$ $5.8 \mathrm{Mpc}$, Schulte-Ladbeck et al. 2001).

The SF regions of Mkn 209 are morphologically reminiscent of those in Mkn 5 (Sect. 4.6), and are dominated by two bright sources ( $a$ and b, Fig. ??a). Gil de Paz et al. (2003, hereafter GMP03) found that the $\mathrm{H} \alpha$ emission peaks at the western SF region a (Fig. ??a). Additional diffuse $\mathrm{H} \alpha$ emission is also present further to the east, close to region $b\left(\approx 18^{\prime \prime}\right.$ from $\left.a\right)$. HST observations of knot a (Deharveng et al. 1994) showed the 

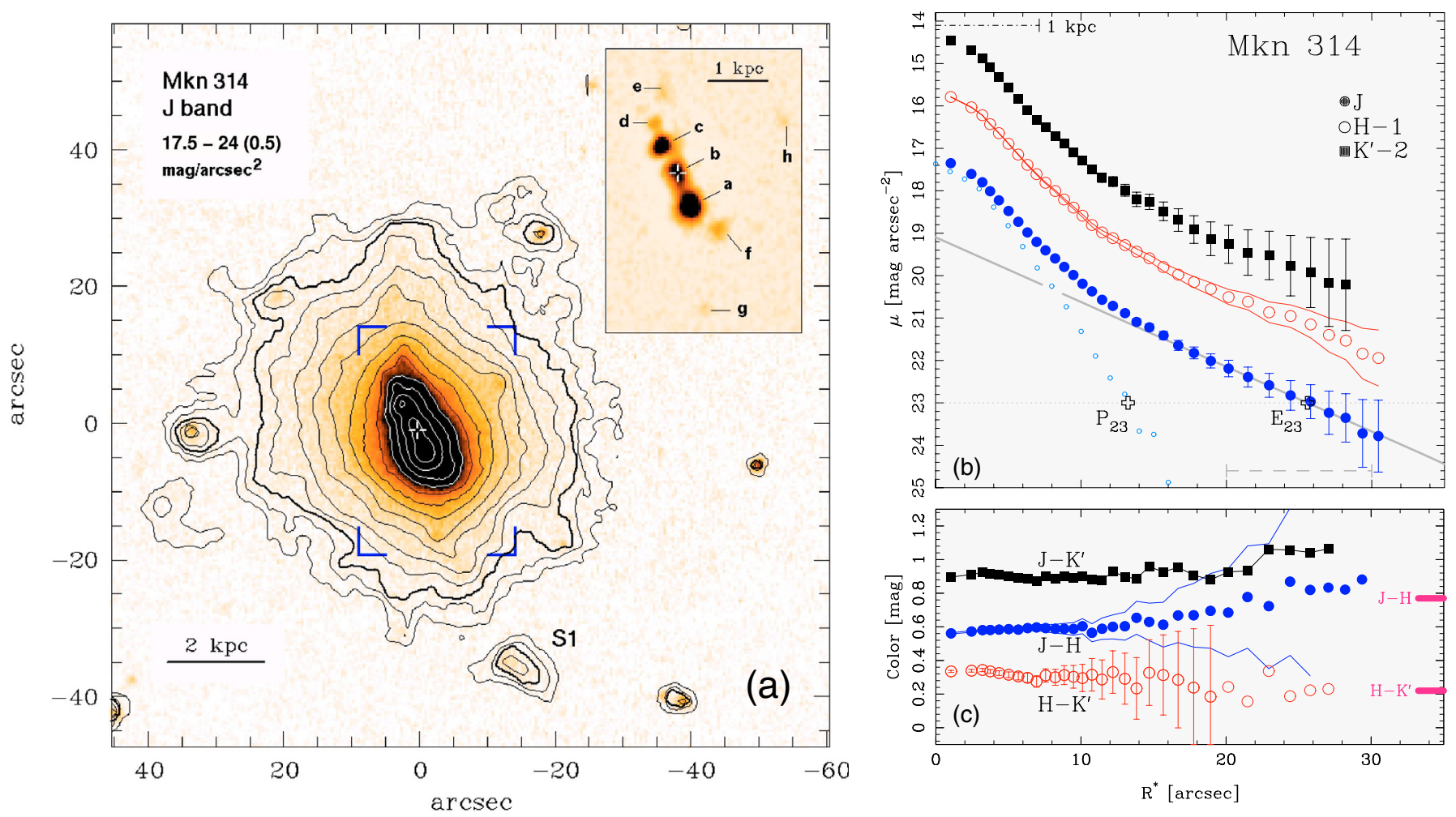

Fig. 1. a) Contours overlaid with a $J$ image of $\mathrm{Mkn} 314(D=28.9 \mathrm{Mpc})$. Contours, corrected for Galactic extinction, go from 17.5 to $24 J \mathrm{mag} / \square^{\prime \prime}$ in steps of $0.5 \mathrm{mag}$. The $23 \mathrm{~J} \mathrm{mag} / \square^{\prime \prime}$ isophote is depicted by the thick contour. The inset shows an unsharp-masked (see Sect. 3.2) and magnified version of the central region of the BCD (indicated by brackets in the contour map image). Compact sources arranged in a bar-like structure along the major axis of the BCD are marked, with the labeling of sources a-c following the denomination by Mazzarella \& Boroson (1993). The bright star-forming region $b$ is marked with a white cross in both the main plot and the inset. The detached SW source S1 shows active star formation. b) Surface brightness profiles (SBPs) of Mkn 314 in the $J, H$ and $K_{\mathrm{s}}$, corrected for galactic extinction. For a better visualization, the $H$ and $K_{\mathrm{s}}$ SBPs are shifted by -1 and -2 mag, respectively. The thick solid line shows an exponential fit to the stellar LSB component in $J$ (cf. Sect. 3), computed within the radius range indicated by the long-dashed bar at the bottom of the diagram. The emission in excess to the fit (small open circles) is attributable to the starburst component, which dominates the light in the inner part of Mkn 314. The isophotal radii $P_{23}$ and $E_{23}$ of the star-forming and LSB component at the surface brightness level of $23 J$ mag/ $\square$ " (horizontal dotted line) are indicated. The bar at the upper left indicates a galactocentric radius of $1 \mathrm{kpc}$. c) Color profiles, computed by subtraction of the SBPs (panel b)). The thick lines at the rightmost part of the diagram indicate the mean $J-H$ and $H-K^{\prime}$ colors of the LSB component (see Sect. 3.1).

young stars to have ages $\leq 12 \mathrm{Myr}$, in agreement with spectral evolutionary synthesis models by Mas-Hesse \& Kunth (1999), who derived a burst age of $\approx 2.7$ Myr. Furthermore, spectroscopic studies of Mkn 209 revealed the presence of Wolf-Rayet stars (Schaerer et al. 1999; Guseva et al. 2000).

The source c (Fig. ??a), detected in optical images $\sim 15^{\prime \prime}$ northeast of $b$, is nearly absent in the NIR. As indicated by its blue colors on uncalibrated optical-NIR color maps, and the presence of a local $\mathrm{H} \alpha$ maximum (GMP03), region c may be strongly affected by nebular emission. The faintness of this source in the NIR may indicate that the stellar population in this area is still too young ( $\lesssim 8 \mathrm{Myr}$ ) to be dominated by Red Super Giant (RSG) stars.

From an IUE UV spectral study, Fanelli et al. (1988) conjectured that Mkn 209 could be a young galaxy, currently undergoing its first episode of star formation. A later HST study by Deharveng et al. (1994) showed, however, that the observed red colors could not be attributed to RSG stars alone, but required the presence of an older stellar population. An old population was confirmed by P96a, who reported almost constant, red ( $B-R \sim 1)$ colors for the extended, elliptical stellar host galaxy of Mkn 209, first detected by LT86. Recent $J$ and $H$ color magnitude diagram studies of Mkn 209, using HST NICMOS data (Schulte-Ladbeck et al. 2001), showed the presence of stars with ages $>1-2$ Gyr, in agreement with the above results.

The SBPs of the LSB host can only be analyzed outside the very extended plateau emission of the starburst, for $R^{*}>18^{\prime \prime}$. An exponential fit, when extrapolated inwards, nowhere implies an intensity brighter than the observed one. It leads, however, to a local depression of the derived radial intensity distribution of the starburst component at $R^{*} \sim 7^{\prime \prime}$. In the $H$ band, an exponential fit results in an unphysical decomposition solution (starburst extent $P_{\text {iso }} \approx 0.02 \mathrm{kpc}$ ). A type V LSB profile, fitted by a med (Eq. (2)) with a cutoff radius $b \alpha \approx 14^{\prime \prime}$, could provide a more plausible decomposition, but cannot be confirmed, due to the numerous fore- and background sources which limit the reliability of the SBPs at their outermost data points $\left(R^{*} \gtrsim 20^{\prime \prime}\right)$. We show a formal decomposition using an exponential fit in the $J$ band only (Fig. ??b and Table 2). 


\section{3. $M k n 996$}

Mkn $996\left(M_{B}=-16.6 \mathrm{mag}\right)$ may be considered a prototypical example of the nE BCD class. Its starburst component is situated near the geometrical center of its smooth LSB host galaxy and shows a very compact morphology, with SF activity being confined to the inner $\approx 3^{\prime \prime}\left(R^{*} \approx 315 \mathrm{pc}\right)$ of the BCD as evidenced by Thuan et al. (1996) using HST WFPC $2 V$ and $I$ data. Optical spectra display strong Wolf-Rayet features, characteristic of WN and WC stars, and imply a low oxygen abundance $\left(Z \approx Z_{\odot} / 10\right)$, as well as an unusually high electron density of $\sim 5 \times 10^{4} \mathrm{~cm}^{-3}$ (Thuan et al. 1996; Schaerer et al. 1999). The HST data by Thuan et al. (1996) revealed dust patches which extend to the north of the central SF region, as well as a central two-arm spiral pattern with a size of $160 \mathrm{pc}$.

The blue colors these authors derive in the vicinity of this central region, $V-I \gtrsim 0.15$, reflect the dominant emission of the young stellar populations and ionized gas, superposed on the old, red LSB component. Interestingly, the NIR color profiles (Fig. ??c) show within their central 2 arcseconds colors that are significantly redder $\left(J-H \approx 0.6, H-K^{\prime} \gtrsim 0.8\right)$ than those expected for young starbursts.

Measurements in $4^{\prime \prime} \times 4^{\prime \prime}$ apertures, i.e. several times larger than the angular resolution of the images, yield $(J-H) \approx 0.61$, $\left(H-K^{\prime}\right) \approx 0.7$ (after correction for the underlying continuum), confirming that these colors are not due to imperfect PSF-equalization during the generation of the color profiles. Such NIR colors cannot be accounted for by stellar and nebular emission alone (see, e.g., Fig. 1 in Campbell \& Terlevich 1984; or Fig. 1 in N03). On the assumption of an internal extinction of $E\left(H-K^{\prime}\right) \sim 0.3 \ldots 0.4 \mathrm{mag}$ and a young $(\tau \sim 5 \mathrm{Myr})$, metalpoor $\left(Z \approx Z_{\odot} / 10\right)$ starburst, such colors could be reproduced. Such an extinction would, however, correspond to a large color excess in the optical, $E(V-I) \sim 1.9 \ldots 2.6 \mathrm{mag}$, in disagreement with the observed blue values of $V-I$. We find that the $V$ and $J$ band intensity maxima coincide within a fraction of an arcsecond, i.e. given the resolution and alignment uncertainties, a significant shift is not found. Color maps suggest increasing extinction only northwest of the region discussed here.

Alternatively, the red $J-H$ starburst colors may be explained by the presence of RSG stars. In metal-poor young stellar populations, the fraction of such sources is higher than at solar metallicities, leading to correspondingly redder $J-H$ values. This effect is not properly reproduced by current stellar evolution models, which therefore may predict too blue $J-H$ colors at low metallicities (cf., e.g., Sect. 4.2 in Vanzi 2003, and references therein ${ }^{2}$ ). If the burst is indeed already in its RSG-dominated phase $(\tau \gtrsim 6.5 \mathrm{Myr})$, then the red $H-K^{\prime}$ colors cannot be attributed to dominant nebular emission any more. Instead, these may originate from blackbody emission of heated (few $100 \mathrm{~K}$ ) dust, contributing mainly to the $K$ band flux and thereby causing red $H-K^{\prime}$ colors (e.g. Campbell \& Terlevich 1984).

\footnotetext{
2 Vanzi (2003) and Origlia et al. (1999) discuss the effect on $J-K$. However, the colors of metal-poor RSGs $(J-H \sim 0.6 \ldots 0.8, H-$ $K \sim 0.1 \ldots 0.2$, Elias et al. 1985 for RSGs in the SMC) imply that an increased frequency of such sources will affect the integral $J-H$ and $J-K$ colors of a young stellar population in a very similar way.
}

An intriguing property of this galaxy, discovered by Thuan et al. (1996), is that is has a population of several globular clusters with an age of $\approx 10 \mathrm{Gyr}$ in the very outskirts of the LSB component, asymmetrically distributed towards the SW of the galaxy, in contrast to the symmetric outer isophotes.

Surface photometry studies by Thuan et al. (1996) showed that for $R^{*} \gtrsim 8^{\prime \prime}$ the stellar LSB host can be well fitted by an exponential law with a scale length $\alpha=420$ pc. The colors of the outer regions of the galaxy $(V-I \approx 0.9)$ are characteristic of an old LSB population. These results are in good agreement with our NIR surface photometry, from which we infer an exponential intensity decrease of the LSB component with a scale length between 400 and 430 pc. Both our results, and those by Thuan et al. (1996) are in contrast with the conclusion by Doublier et al. (1999), that Mkn 996 follows an overall de Vaucouleurs-profile. This disagreement might stem from a subtle excess for $R^{*}>18^{\prime \prime}$, visible in both our SBPs and those by Thuan et al. (1996), which at first glance points to a shallow, more extended LSB population. We believe, however, that this small excess originates from the combined emission of the asymmetrically distributed globular cluster population (see above). These sources cannot be properly resolved and subtracted using ground-based data, and could therefore induce a slight flattening of the SBPs at large radii. We therefore fitted the SBPs for $R^{*}<18^{\prime \prime}$ in $J$ only, i.e. at radii where the emission can be reliably attributed to the unresolved stellar LSB population of Mkn 996.

\subsection{Mkn 370 (NGC 1036, UGC 02160)}

Compared to other BCDs, the iE-classified (LT86) BCD Mkn 370 is relatively metal-rich $\left(\mathrm{Z} \approx Z_{\odot} / 2, M_{B}=-17.1\right)$. Deep broad-band surface photometry in the optical and highresolution color and $\mathrm{H} \alpha$ maps were first presented in $\mathrm{C} 01 \mathrm{a}, \mathrm{b}$, and a dedicated spectrophotometric study of this BCD has recently been published in Cairós et al. (2002).

Two major sources, separated by $\approx 7^{\prime \prime}(380 \mathrm{pc}$ ), are located in the central region of the galaxy. These correspond to the "double nucleus" catalogued in Mazzarella \& Boroson (1993) and are labeled a and b in Fig. ??a, following the nomenclature of the latter authors. As apparent from the contrast-enhanced inset in the $J$ band image (Fig. ??a), the elongated central region a splits into a brighter southwestern and a fainter northeastern source, similar to what was reported by Nordgren et al. (1995). The NIR images show several fainter sources, roughly aligned with the major axis of region $a$.

The positions of both $a$ and $b$ coincide in the optical and in the NIR, although $b$, which is a conspicuous source in the blue, is only weakly detected in the NIR. The blue colors of this source $(U-B=-0.79)$, together with its emission line spectrum, flat continuum and the large $\mathrm{H} \alpha$ equivalent width $(E W(\mathrm{H} \alpha) \approx 500 \AA$; Nordgren et al. 1995; Cairós et al. 2002) are indicative of a young stellar population (ages $\leq 5 \mathrm{Myr}$ ) and substantial ionized gas contribution. Knot a presents slightly redder colors $(U-B=-0.60)$ and a high continuum level, with absorption features that witness a substantial underlying population of older stars. 
The finding that knot $\mathrm{b}$ shows no appreciable old stellar background in NIR images, but is dominated by nebular emission, indicates that the double-nucleus morphology of Mkn 370 is attributable to extranuclear SF activity in knot $b$.

The LSB population, traceable on our images out to $R^{*} \approx$ 50 ", provides about $80 \%$ of the $J$ light. Its NIR colors (Table 3 ), as well as the integrated $B-J$ color of 2.3 mag, computed using data from Cairós et al. (2002), are both consistent with an old stellar population. In NIR wavelengths the LSB component shows a roughly exponential slope, with a possible, but not significant slight flattening for $R^{*} \lesssim 30^{\prime \prime}$. The $J$ band scale length, $\alpha=0.64 \mathrm{kpc}$, is significantly smaller than the $\alpha \sim 1 \mathrm{kpc}$ inferred from $B$ band data (Cairós et al. 2002). This difference is most likely due to the multiple extranuclear $\mathrm{H} \alpha$-emitting sources, distributed roughly along the optical major axis of Mkn 370 (Nordgren et al. 1995; Cairós et al. 2002). Similar to what is discussed for Mkn 600 (Sect. 4.7), these sources may contaminate the LSB emission at large radii especially in optical wavelengths, artificially increasing the scale length of the old population.

\subsection{Zw 115 (UGC 09893)}

This iI - classified (LT86) BCD $\left(M_{B}=-16.4\right)$ was described in the Atlas of interacting galaxies by Vorontsov-Vel'Yaminov (1977) as "a pair of coalescents". Optical surface photometry for this system was first presented in P96a, while further $B, R$ and $\mathrm{H} \alpha$ images have recently been published in the catalogue of Blue Compact Dwarf Galaxies by GMP03.

I Zw 115 presents a peculiar morphology, displaying several intensity maxima within an LSB component which, other than in the majority of BCDs, shows boxy outer isophotes. P96a conjectured that this could be a signature of a dynamically unrelaxed underlying stellar component. The derived $B-$ $J$ color of the LSB host galaxy, $\sim 1.8 \pm 0.3 \mathrm{mag}$, is consistent with an age of a few Gyr. The brightest region, which spatially coincides in the optical and NIR broad-band frames, is located towards the south-west of the LSB component and is resolved into two smaller regions in the NIR images, labeled $A$ and B in Fig. ??. The latter regions seem to delineate, together with several other condensations, a ring-like structure (" $R$ " in Fig. ??a) with a projected diameter of $\sim 500 \mathrm{pc}$ in the southeastnorthwest direction. Northeast of this ring-like structure, several maxima are detected, reminiscent of the tails of SF regions observed in "cometary" BCDs. However, $\mathrm{H} \alpha$ emission in I Zw 115 is primarily confined to one single SF region, which is displaced $\sim 15^{\prime \prime}$ northeastwards with respect to $a$ and $b$ (see the maps by GMP03).

The SBPs show an exponential decay at large radii, with a $J$ band scale length of $\approx 530 \mathrm{pc}$, compatible to the one derived from optical data (P96a, transformed to the distance adopted in this paper). The type V LSB profile detected by the latter authors is confirmed by our analysis, which, however, implies a stronger central depression $q$ and larger cutoff radius $b \alpha$ than those inferred in the optical. Using the approach described in Sect. 3, we can constrain the parameters of a med to $(b, q) \approx 2.4,0.80$.

\subsection{Mkn 5 (UGCA 130)}

While Mkn 5 can be considered an average BCD with respect to its absolute magnitude $\left(M_{B}=-15.7\right)$, oxygen abundance $\left(\sim Z_{\odot} / 7\right.$; Izotov \& Thuan 1999) and the regular, elliptical isophotes of its LSB host galaxy, it displays a peculiar, "cometary" morphology of its SF regions. This latter classification (LT86) denotes the presence of a dominant SF complex ("A" in Fig. ??a) towards one end of an elongated stellar host galaxy, with fainter SF regions distributed along the optical major axis. However, the "iI,C" BCDs, selected by this morphology of the SF regions, typically display a more elongated, irregular host galaxy than that observed in Mkn 5 (see Noeske et al. 2000).

The dominant SF region A shows WR features (Conti 1991) and is the locus of intense and moderately extended nebular emission $(E W(\mathrm{H} \alpha) \gtrsim 60 \AA$; Noeske 1999). The colors of this knot $(U-B=-0.78, B-V=0.49, V-R=0.18, V-I=0.19$ within a $4^{\prime \prime}$ aperture) are compatible with a burst age $\leq 4 \mathrm{Myr}$ (Cairós 2000). Note that $B-V$ appears relatively red due to the contribution of nebular line emission.

Among the SF regions forming the "tail" northwards of A, source C (Fig. ??a) is the brightest in the NIR, in contrast to the optical images, where this knot is only marginally detected. The second-brightest source in the optical, B, situated $\sim 20^{\prime \prime}$ north of $A$, is barely seen even on contrast-enhanced $J$ images. The $\mathrm{H} \alpha$ equivalent width at this position is relatively low, $\sim 15 \AA$, suggesting that its blue colors are due to a young stellar population, rather than strong nebular emission.

The NIR surface brightness profiles reveal an extended plateau feature at high to intermediate intensity levels, similar to optical profiles (C01a). The exponential LSB profile, observed in the outskirts of Mkn 5, flattens for small radii and can be well approximated by a med with $(b, q)=1.4,0.65$. The $J$ band exponential scale length, $\alpha \approx 5^{\prime \prime}(0.37 \mathrm{kpc})$, is in good agreement with the results by Noeske et al. (2001a) and C01a.

\subsection{Mkn 600}

Star-forming activity in this $\mathrm{iE} \mathrm{BCD}\left(M_{B}=-15.5, \mathrm{C} 01 \mathrm{~b}\right.$; $12+\log \mathrm{O} / \mathrm{H}=7.83$, Izotov \& Thuan 1999; Cairós 2000) occurs predominantly in its inner part $\left(R^{*} \leq 8^{\prime \prime}\right)$, where two distinct maxima, A and B, are seen in optical images (C01b). In the NIR images, each of the latter regions is resolved into two condensations, respectively denoted $A 1, A 2$ and $B 1, B 2$ in Fig. ??a. The ongoing SF activity in both $A$ and $B$ is indicated by deep $\mathrm{H} \alpha$ images ( $\mathrm{C} 01 \mathrm{~b}$ ) which show that both regions coincide with local peaks in $\mathrm{H} \alpha$ line intensity and $\mathrm{H} \alpha$ equivalent width maps. Both colors and $\mathrm{H} \alpha$ equivalent widths of $\mathrm{A}$ and $B$ point to burst ages $<5 \mathrm{Myr}$ (Cairós 2000). At fainter surface brightness levels, optical and NIR data reveal a regular LSB host galaxy which extends out to $R^{*} \sim 30^{\prime \prime}$ and displays roughly elliptical isophotes. The colors of the latter component ( $V-R \approx 0.3, V-I \approx 0.8$, C01a; for NIR colors, see Table 3 ) are indicative of a several Gyr old stellar population.

A chain of fainter knots extends southeastwards from the center of Mkn 600, and connects with a moderately bright, $\mathrm{H} \alpha$-emitting source (region c in Fig. ??). Source c, marginally 
detected in the NIR frames, emits strongly in $\mathrm{H} \alpha(E W(\mathrm{H} \alpha) \approx$ $590 \AA$ ) , and presents colors slightly bluer than the central knots ( $U-B=-0.64,-0.72$ and -0.86 for knots $\mathrm{a}, \mathrm{b}$ and $\mathrm{c}$, respectively), suggesting a possible propagation of SF activity.

The hypothesis that the peculiar distribution of SF activity in Mkn 600 may be the result of an ongoing or recent interaction cannot be discarded, given the presence of a nearby H I companion (Taylor et al. 1993). In our NIR images we detect no counterpart of this object down to a surface brightness limit of $\approx 23 \mathrm{~J} \mathrm{mag} / \square^{\prime \prime}$.

As is evident from Fig. ??a, the influence of the aforementioned extranuclear SF region $\mathrm{C}$ is relatively small in the NIR. This is also the case for other condensations in the vicinity of $c$ (circles in Fig. ??a). Surface brightness profiles, derived after subtraction of these sources, do therefore not differ notably from those derived from the original images. For $R^{*}>9$ arcsec, the SBPs were approximated by an exponential distribution with scale lengths $\alpha \approx 4.8^{\prime \prime}$, slightly smaller than those derived by $\mathrm{C} 01$ a from optical data $\left(\alpha \approx 5^{\prime \prime} .5\right)$. This difference is probably due to the stronger contribution of region $\mathrm{C}$ in optical bands (see also Sect. 4.4).

The integral colors we infer for Mkn 600 can hardly be reconciled with those by Doublier et al. (2001). These authors report an integral $J-K$ color of $\sim 1 \mathrm{mag}$, much redder than the $J-K$ of 0.65 we derive.

\subsection{NGC 6789 (UGC 11425)}

NGC 6789, an intrinsically faint $\left(M_{B}=-14.3\right.$, Drozdovsky et al. 2001) galaxy with an iE morphology, is located at a distance of about 3.6 Mpc (Drozdovsky et al. 2001) and therefore belongs to the most nearby BCD candidates known to date, together with IC 10 (Richer et al. 2001) and IC 4662 (N03a). Karachentsev \& Makarov (1998) found NGC 6789 to be very isolated, situated in the local void.

Deep optical imaging (Drozdovsky \& Tikhonov 2000) of this system revealed properties typical among BCDs, in particular an inner $\left(R^{*} \sim 150 \mathrm{pc}\right)$, high surface-brightness ionizing stellar population within a red $(V-I \approx 0.9 \mathrm{mag})$ underlying LSB host, extending out to at least $0.6 \mathrm{kpc}$.

Subsequent HST/WFPC2 observations (Drozdovsky et al. 2001) made it possible to resolve this galaxy into more than 15000 stars, and to derive a minimum age of $1 \mathrm{Gyr}$ from color-magnitude diagrams, in agreement with the red $V-I$ LSB colors.

Inspection of the $J$ SBP slope shows that the LSB emission of NGC 6789 follows a type V profile, which was fitted by a med with a cutoff radius of $3.3 \alpha$ and central depression of $q \approx$ 0.7 . The scale length we derive in the outer regions, $\alpha \approx 200 \mathrm{pc}$ (Table 2), differs from that inferred by Drozdovsky \& Tikhonov (2000) $(\alpha \approx 280 \mathrm{pc}$ ). Since the moderately flattening type $\mathrm{V}$ SBP of the LSB host was not revealed by optical data, the latter authors applied an exponential fit to the entire LSB emission (for $R^{*} \gtrsim 20^{\prime \prime}$ ), thereby including slightly flattened parts of the SBP inside the cutoff radius ( $b \alpha \approx 38^{\prime \prime}$ ). The exponential part of the med fitted to our $J$ band data has been adjusted to the steeper, outer exponential regime of the profile $\left(R^{*}>39^{\prime \prime}\right)$.
The exponential scale lengths and extrapolated central surface brightnesses inferred for NGC 6789, both by Drozdovsky \& Tikhonov (2000) and in this paper (see Table 2), indicate a compact structure of the stellar host. This further supports the view that NGC 6789 displays properties typical of BCDs, rather than those of more diffuse dwarf irregulars.

\subsection{Mkn 324 (UGCA 439)}

Mkn $324\left(M_{B}=-16.5\right)$ is a compact iE BCD which displays two distinct $\mathrm{SF}$ regions close to its geometrical center, referred to by Mazzarella \& Boroson (1993) as a and b (Fig. ??a) and studied by the same authors.

Surface photometry in the optical has been previously presented by Doublier et al. $(1997,1999)$ and C01a. The latter authors (C01b) also computed optical color maps, and disclosed in deep $\mathrm{H} \alpha$ images a complex morphology of the ionized gas, most notably a large supershell expanding to the northwest of the starburst region. The $\mathrm{H} \alpha$ intensity was found to peak in between the pair of bright SF regions a and b (C01b, Petrosian et al. 2002). In its LSB periphery, Mkn 324 is delimited by regular outer isophotes, and shows no conspicuous signatures of a strong gravitative perturbation.

It appears nevertheless possible that the starburst activity of this system has been triggered by a distant low-mass companion. High-resolution H I VLA maps (van Zee et al. 2001) have recently unveiled a gas cloud with a mass of $\sim 2 \times 10^{8} M_{\odot}$, approximately $115 \mathrm{kpc} \mathrm{NW}$ of the BCD (values transformed to the distance adopted in this paper), with a projected velocity difference of $\sim 100 \mathrm{~km} \mathrm{~s}^{-1}$ to Mkn 324. A follow-up analysis of this system by Cairós et al. (2004, in prep.) has revealed an intrinsically faint, low-surface-brightness optical counterpart to this H I source. In this respect, it appears noteworthy that the H I component of Mkn 324 shows an overall solid-body rotation, though with conspicuous kinematical peculiarities, reminiscent of tidal tails (van Zee et al. 2001). 2D studies of the ionized gas component of the BCD (Petrosian et al. 2002) have shown a largely chaotic velocity field, with some underlying regular pattern.

Our surface photometry reveals in all NIR bands an exponential LSB distribution in the radius range $5^{\prime \prime} .9 \lessgtr R^{*} \lesssim 11^{\prime \prime}$, with a moderately strong starburst emission. For this outer LSB component, we derive an exponential $J$ band scale length of $\approx 280 \mathrm{pc}$, in agreement with the $I$ band scale length derived by C01a. Some excess emission above the exponential SBPs for $R^{*}>11^{\prime \prime}$ is presumably due to imperfect subtraction of diffuse sources adjacent to the eastern edge of the LSB component (sources E1 and E2 in Fig. ??a).

The possibility that this excess above the exponential fit at large radii is a signature of a Sérsic law with an exponent $\eta \gtrsim 1$ could be dismissed. Sérsic fits over different radii in the interval $5^{\prime \prime} \cdot 9 \leq R^{*} \leq 15^{\prime \prime}$, yielding exponents $\eta$ between 1.4 and 2, could not be extrapolated inwards without exceeding the observed SBPs at small radii, i.e., they fall short of describing the SBP of the LSB component. 


\subsection{Mkn 450 (UGC 08323)}

Star-forming regions within this $\mathrm{iE} \mathrm{BCD}$ are distributed over a large surface fraction of its elliptical stellar LSB host. Individual condensations appear to be arranged within 3 distinct associations. Close to the geometrical center of the LSB host, an elongated structure (c2 in Fig. ??) with an extent of roughly $2 \mathrm{kpc}$ is seen along the southeast-northwest direction. It is displaced relative to the optical major axis of the galaxy, which roughly runs in the east-west direction. Adjacent to the southwestern edge of c2, a fainter complex of knots, c3, can be discerned. A third strip of SF knots, c1, is seen NW of regions $\mathrm{c} 2$ and $\mathrm{c} 3$, oriented roughly perpendicular to the former. The latter feature hosts the brightest individual condensations, $\mathrm{a}$ and $\mathrm{b}$ in Fig. ??a. The photometric properties of each source (a: $m_{J} \sim 17 \mathrm{mag}, J-H \sim 0.3 \mathrm{mag}, H-K^{\prime} \sim 0.6 \mathrm{mag}$; b: $\left.m_{J} \sim 18 \mathrm{mag}, J-H \sim 0.2 \mathrm{mag}, H-K^{\prime} \sim 0.6 \mathrm{mag}\right)$, derived after correction for the surrounding continuum, point to young stellar populations and strong contributions of ionized gas. Recent $\mathrm{H} \alpha$ maps by GMP03 confirm that SF activity in Mkn 450 is largely confined to regions a and b, while only faint $\mathrm{H} \alpha$ emission is present at the location of the features $\mathrm{c} 2$ and $\mathrm{c} 3$.

The alignment of the morphological complex c1 with respect to the LSB host is atypical among $\mathrm{iE} \mathrm{BCDs;} \mathrm{usually,}$ such elongated sequences of SF regions are found closer to the centers of such objects, at position angles parallel rather than perpendicular to the major axis of the LSB host (see e.g. the morphological catalogs by Telles et al. 1997; C01a; GMP03). Mkn 450 is a field BCD (Popescu et al. 1999) which shows no peculiarities among its other properties, such as its $B$ magnitude of $M_{B}=-16.70$ (Vennik et al. 2000), and both a moderate metal-deficiency $\left(1 / 5 Z_{\odot}\right.$, Garnett 1990) and star formation rate $\left(\sim 0.1 M_{\odot} \mathrm{yr}^{-1}\right.$; Popescu et al. 1999 , value transformed to the distance adopted in this paper).

Subtraction of only the brightest irregular SF regions reveals a modest central flattening of the stellar LSB host galaxy, well described by a med with $(b, q)=(1.5,0.65)$.

\subsection{NGC 5058 (UGC 08345, MKN 786)}

Deep images of this galaxy show two maxima (Fig. ??a) within a common, extended LSB component, which at low surface brightness levels displays an asymmetric, curved morphology (see the $25.5 R \mathrm{mag} / \square^{\prime \prime}$ isophote in Fig. ??a).

NGC 5058 was previously classified as a galaxy pair (KPG 370; Karachentsev 1972). The components of this pair (N and S, Tifft 1982) are most likely identical to the maxima visible in Fig. ??a, as can be inferred from their relative positions (projected separation $8 . .7$ at a position angle $12^{\circ}$; Tifft 1982 for components $\mathrm{N}$ and S). We therefore adopted the latter denomination (cf. the inset in Fig. ??a).

On contrast-enhanced NIR images, source $\mathrm{N}$ resolves into 3 distinct regions (N1, N2, N3 in Fig. ??a). Fainter extensions emanate from this central source to the north, and southwards, connecting with source S. A third extension ("NW" in Fig. ??a) extends northwestwards from the southern source S. New $\mathrm{H} \alpha$ images by GMP03 show that the SF activity peaks close to source $\mathrm{S}$, and around a northeastern component, detached from the central regions ("NE" in Fig. ??). The same data reveal only weak $\mathrm{H} \alpha$ emission along the NW extension, suggesting that this feature is not mainly due to nebular emission.

Since NGC 5058 is located at a small angular separation (11.6) from the center of the Virgo Cluster, redshift-based distance determinations using Virgocentric infall models suffer from the Triple Value Problem (Teerikorpi et al. 1992). An alternative distance determination by the same authors, based on the Tully-Fisher relation, yields a large distance of $37.7 \mathrm{Mpc}$, even outside the range of values allowed within the uncertainties of the Triple Value Problem (see Fig. 1 in Teerikorpi et al. 1992). We suggest that the HI velocity width that these authors use, $118 \mathrm{~km} \mathrm{~s}^{-1}$, may be partly enlarged by the southern component $\mathrm{S}$, which, by its velocity difference to component $\mathrm{N}$ $\left(58 \mathrm{~km} \mathrm{~s}^{-1}\right.$, Tifft 1982), might be a kinematically distinct subunit of NGC 5058.

Dynamical distortions or even a past merger event in this galaxy appear also possible in view of the peculiar shape of the isophotes in the LSB regime, as mentioned above. Irregularities south of the center of the galaxy are traceable down to the limits of the $J$ band data $\left(\sim 24 J \mathrm{mag} / \square^{\prime \prime}\right)$. These condensations in the southernmost outskirts of the galaxy show no obvious $\mathrm{H} \alpha$ emission (GMP03).

We adopt here a distance $D=11.6 \mathrm{Mpc}$, using the velocity of NGC 5058 with respect to the center of the Virgo Cluster (see N03) and $H_{0}=75 \mathrm{~km} \mathrm{~s}^{-1} \mathrm{Mpc}^{-1}$; note that also for much larger distances, up to $D \lesssim 31.1 \mathrm{Mpc}$, the resulting absolute magnitude would be $>-18 \mathrm{~B}$ mag, still qualifying NGC 5058 as a BCD.

In view of the complex morphology at all surface brightness levels, and of wide-spread SF activity and further condensations, a decomposition of the surface brightness profiles is rendered problematic. For $R^{*}>21.6^{\prime \prime}$ i.e. outside strong irregular emission in the $J$ images, the SBPs can be approximated by an exponential, albeit with some systematic differences (Fig. ??b). The SBPs suggest however the presence of a type V SBP at large radii. An example decomposition by means of a med is shown in Fig. ??b. The parameters of this function cannot be reliably constrained, given the extended influence of irregular and starburst emission, and the low $S / N$ levels $\left(\mu_{J} \sim 22 \ldots 23 \mathrm{mag} / \square^{\prime \prime}\right)$ at which we suspect the flattening occurs. The results of the profile decomposition given in Table 2 therefore refer to the exponential fit to the LSB host.

\section{Summary}

This work is part of a series of papers that describe an imaging study of a large sample of Blue Compact Dwarf (BCD) galaxies in the NIR. We have presented deep $J, H$ and $K^{\prime}$ NIR images for 11 northern BCDs. These data, together with those presented in N03a and C03a, constitute the largest sample of BCDs analyzed so far in the NIR. Hitherto unreached deep surface brightness limits $\left(23 \ldots 24 J \mathrm{mag} / \mathrm{arcsec}^{2}\right)$ allow detailed surface photometry of the evolved, extended stellar host galaxies of such objects, to derive the structural parameters of their light distributions and their colors. 
Radial surface brightness profiles (SBPs) were decomposed into the contributions of the extended stellar host galaxy, and of the starburst component, the latter encompassing the emission of current star-forming activity and recently formed young stars. This task was accomplished through the extrapolation of fits to the SBPs of the host galaxy, at larger radii where the emission of the centrally concentrated starburst component vanishes. In the NIR, the fractional contribution of the starburst is lower than in the optical, making it possible to study the host galaxies closer to their center, and to test for central deviations from the exponential distributions that the host galaxy SBPs typically display in their outer parts.

For each object we present $J$ contour maps, overlaid with the $J$ images and the derived surface brightness and color profiles. A detailed description of the morphology and peculiar properties of each galaxy are also provided.

The results of this work support the general findings described for BCDs in the NIR by N03a, and can be summarized as follows:

1. At larger galactocentric radii, the NIR SBPs of the evolved stellar host galaxies show a roughly exponential decay, in agreement with previous results from optical studies. Exponential scale lengths derived in the optical and NIR are mostly compatible within the uncertainties, indicating that old stellar components of BCDs typically have no appreciable color gradients at intermediate and large radii.

2. In 4 out of 11 sample BCDs, the SBP of the host galaxy shows a central flattening with respect to its exponential decay at larger radii, typically within $R^{*} \gtrsim 2-3$ exponential scale lengths of the outer profile. In 4 additional objects, such inwards-flattening exponential SBPs (denoted "type V" in Binggeli \& Cameron 1991) of the stellar host galaxies are found to be likely. These results support our previous notion (N03) that centrally flattening SBPs may be frequent in stellar hosts of BCDs, but largely remain undetected at optical wavelengths where central starburst emission is more dominant.

3. The fact that NIR data are less affected by nebular emission and dust extinction than optical data, yet more susceptible to emission of hot dust, allows a more profound interpretation of the morphology and properties of individual sources of the star-forming regions. Such a less biased view of the spatially resolved star formation history of BCDs may be essential for understanding the probably complex mechanisms that govern their massive, spatially extended starforming activity.

Acknowledgements. Research by K.G.N. has been supported by the Deutsche Forschungsgemeinschaft (DFG) grants FR325/50-1 and FR325/50-2. P.P. and K.J.F. received support from the Deutsches Zentrum für Luft- und Raumfahrt e.V. (DLR) under grant 50 OR 9907 7. L.M.C. acknowledges support from the European Community Marie Curie Grant HPMF-CT-2000-00774. The authors wish to thank the staff of the German-Spanish Astronomical Center at Calar Alto for their friendly support during the observations, and the referee of this paper, Dr. G. Comte, for his helpful comments. We are indebted to Dr. Tom Jarrett for kindly providing photometric data from the 2MASS survey prior to publication, which allowed us to calibrate a part of the present sample. We thank Dr. Uta Fritze - v. Alvensleben, P. Anders, J. Bicker and J. Schulz for kindly providing the GALEV models. This research has made use of the NASA/IPAC Extragalactic Database (NED) which is operated by the Jet Propulsion Laboratory, CALTECH, under contract with the National Aeronautic and Space Administration. This publication makes use of data products from the Two Micron All Sky Survey, which is a joint project of the University of Massachusetts and the Infrared Processing and Analysis Center/California Institute of Technology, funded by the National Aeronautics and Space Administration and the National Science Foundation.

\section{References}

Binggeli, B., \& Cameron, L. M. 1991, A\&A, 252, 27

Cairós, L. M 2000, Ph.D. Thesis, Universidad de La Laguna

Cairós, L. M., Vílchez, J. M., González Pérez, J., Iglesias-Páramo, J., \& Caon, N. 2001a, ApJS, 133, 321 (C01a)

Cairós, L. M., Caon, N., Vílchez, J. M., González-Pérez, J. N., \& Muñoz-Tuñón, C. 2001b, ApJS, 136, 393 (C01b)

Cairós, L. M., Caon, N., García-Lorenzo, B., Vílchez, J. M., \& Muñoz-Tuñón, C. 2002, ApJ, 577, 164

Cairós, L. M., Caon, N., Papaderos, P., et al. 2003, ApJ, 593, 312 (C03)

Campbell, A. W., \& Terlevich, R. 1984, MNRAS, 211, 15

Cannon, J. M., Skillman, E. D., Garnett, D. R., \& Dufour, R. J. 2002, ApJ, 565, 931

Cardelli, J. A., Clayton, G. C., \& Mathis, J. S. 1989, ApJ, 345, 245

Conti, P. S. 1991, ApJ, 377, 115

Cutri, R. M., et al. 2000, The 2MASS Explanatory Supplement

Davies, J. I., \& Phillipps, S. 1988, MNRAS, 233, 553

Deeg, H.-J., Duric, N., \& Brinks, E. 1997, A\&A, 323, 323

Deharveng, J.-M., Albrecht, R., Barbieri, C., et al. 1994, A\&A, 288, 413

Dekel, A., \& Silk, J. 1986, ApJ, 303, 39

Dekel, A., \& Woo, J. 2003, MNRAS, 344, 1131

de Vaucouleurs, G., de Vaucouleurs, A., Corwin, H. G., et al. 1991, Third Reference Catalogue of bright Galaxies (Springer Verlag)

De Young, D. S., \& Heckman, T. M. 1994, ApJ, 431, 598

Doublier, V., Comte, G., Petrosian, A., Surace, C., \& Turatto, M. 1997, A\&AS, 124, 405

Doublier, V., Caulet, A., \& Comte, G. 1999, A\&AS, 138, 213

Doublier, V., Caulet, A., \& Comte, G. 2001, A\&A, 367, 33

Drozdovsky, I. O., Schulte-Ladbeck, R. E., Hopp, U., Crone, M. M., \& Greggio, L. 2001, ApJ, 551, L135

Drozdovsky, I., \& Tikhonov, N. 2000, A\&AS, 142, 347

Elias, J. H., Frogel, J. A., \& Humphreys, R. M. 1985, ApJS, 57, 91

Fanelli, M. N., O'Connell, R. W., \& Thuan, T. X. 1988, ApJ, 334, 665

Garnett 1990

Gerola, H., Seiden, P., \& Schulmann, L. 1980, ApJ, 242, 517

Gil de Paz, A., Zamorano, J., Gallego, J., \& Domínguez, F. de B. 2000a, A\&AS, 145, 377

Gil de Paz, A., Zamorano, J., \& Gallego, J. 2000b, A\&A, 361, 465

Gil de Paz, A., Madore, B. F., \& Pevunova, O. 2003, ApJS, 147, 29 (GMP03)

Guseva, N. G., Izotov, Y. I., Papaderos, P., et al. 2001, A\&A, 378, 756

Guseva, N. G., Izotov, Y. I., \& Thuan, T. X. 2000, ApJ, 531, 776

Izotov, Y. I., \& Thuan, T. X. 1999, ApJ, 511, 639

Jarrett, T. H., Chester, T., Cutri, R., Schneider, S., \& Huchra, J. 2000, AJ, 119, 2498

Karachentsev, I. D., \& Makarov, D. I. 1998, A\&A, 331, 891

Karachentsev, I. D. 1972, Isolated Pairs of Galaxies Catalogue, 7, 3 
Krüger, H., Fritze-v. Alvensleben, U., \& Loose, H.-H. 1995, A\&A, 303, 41

Lin, D. N. C., \& Faber, S. M. 1983, ApJ, 266, L21

Loose, H.-H., \& Fricke, K. J. 1982, in The Most Massive Stars, ESO workshop proceedings, ed. S. D’Odorico, D. Baade, \& K. Kjaer, Garching: European Southern Observatory (ESO), 269

Loose, H. H., \& Thuan, T. X. 1986, Star Forming Dwarf Galaxies and Related Objects, 73 (LT86)

Mac Low, M., \& Ferrara, A. 1999, ApJ, 513, 142

Makarova, L. N., Karachentsev, I. D., \& Georgiev, T. B. 1997, Astron. Lett., 23, 378

Marlowe, A. T., Meurer, G. R., \& Heckman, T. M. 1999, ApJ, 522, 183

Mas-Hesse, J. M., \& Kunth, D. 1999, A\&A, 349, 765

Mazzarella, J. M., \& Boroson, T. A. 1993, ApJS, 85, 27

Noeske, K. G. 1999, Diploma Thesis, Universität Göttingen

Noeske, K. G., Guseva, N. G., Fricke, K. J., et al. 2000, A\&A, 361, 31

Noeske, K. G., Iglesias-Páramo, J., Vílchez, J. M., Papaderos, P., \& Fricke, K. J. 2001a, A\&A, 371, 806

Noeske, K. G., Cairós, L. M., Papaderos, P., Vílchez, J. M., \& Fricke, K. J. 2001b, Ap\&SS, 276, 901

Noeske, K. G., Papaderos, P., Cairós, L. M., \& Fricke, K. J. 2003, A\&A, 410, 481 (N03)

Nordgren, T. E., Helou, G., Chengalur, J. N., Terzian, Y., \& Khachikian, E. 1995, ApJS, 99, 461

Origlia, L., Goldader, J. D., Leitherer, C., Schaerer, D., \& Oliva, E. 1999, ApJ, 514, 96

Papaderos, P. 1998, Ph.D. Thesis, University of Göttingen

Papaderos, P., Loose, H.-H., Thuan, T. X., \& Fricke, K. J. 1996a, A\&AS, 120, 207 (P96a)

Papaderos, P., Loose, H.-H., Fricke, K. J., \& Thuan, T. X. 1996b, A\&A, 314, 59 (P96b)

Papaderos, P., Izotov, Yu. I., Noeske, K. G., Thuan, T. X., \& Fricke, K. J. 2001, in Dwarf Galaxies and their Environment, ed. K. S. de Boer, R. J. Dettmar, \& U. Klein (Shaker Verlag), 111
Papaderos, P., Izotov, Yu. I., Thuan, T. X., et al. 2002, A\&A, 393, 461 Petrosian, A. R., Movsessian, T., Comte, G., Kunth, D., \& Dodonov, S. 2002, A\&A, 391, 487

Popescu, C. C., Hopp, U., \& Rosa, M. R. 1999, A\&A, 350, 414

Richer, M. G., Bullejos, A., Borissova, J., et al. 2001, A\&A, 370, 34

Salzer, J. J., McAlpine, G. M., \& Boroson, T. A. 1989b, ApJS, 70, 479

Schaerer, D., Contini, T., \& Pindao, M. 1999, A\&AS, 136, 35

Schlegel, D. J., Finkbeiner, D. P., \& Davis, M. 1998, ApJ, 500, 525

Schulte-Ladbeck, R. E., Hopp, U., Greggio, L., Crone, M. M., \& Drozdovsky, I. O. 2001, AJ, 121, 3007

Silich, S., \& Tenorio-Tagle, G. 2001, ApJ, 552, 91

Taylor, C. L., Brinks, E., Pogge, R. W., \& Skillman, E. D. 1994, AJ, 107, 971

Taylor, C., Brinks, E., \& Skillman, E. D. 1993, AJ, 105, 128

Telles, E., Melnick, J., \& Terlevich, R. 1997b, MNRAS, 288, 78

Teerikorpi, P., Bottinelli, L., Gouguenheim, L., \& Paturel, G. 1992, A\&A, 260, 17

Thuan, T. X., \& Martin, G. 1981, ApJ, 247, 823

Thuan, T. X. 1985, ApJ, 299, 881

Thuan, T. X., Izotov, Y. I., \& Lipovetsky, V. A. 1996, ApJ, 463, 120

Tifft, W. G. 1982, ApJS, 50, 319

Tully, R. B. 1988, Nearby Galaxies Catalog (Cambridge University Press)

van Driel, W., Arnaboldi, M., Combes, F., \& Sparke, L. S. 2000, A\&AS, 141, 385

van Zee, L., Salzer, J. J., \& Skillman, E. D. 2001, AJ, 122, 121

van Zee, L., Skillman, E. D., \& Salzer, J. J. 1998, AJ, 116, 1186

Vanzi, L. 2003, A\&A, 408, 523

Vennik, et al. 2000

Viallefond, F., \& Thuan, T. X. 1983, ApJ, 269, 444

Vorontsov-Vel'Yaminov, B. A. 1977, A\&AS, 28, 1

Walter, F., Brinks, E., Duric, N., \& Klein, U. 1997, AJ, 113, 2031

Whitmore, B. C., Lucas, R. A., McElroy, D. B., et al. 1990, AJ, 100, 1489 